\title{
A direct role for vitamin D-binding protein in the pathogenesis of COPD?
}

\section{Sarah Dimeloe, Catherine Hawrylowicz}

Epidemiological studies support the importance of adequate vitamin $\mathrm{D}$ status for the maintenance of pulmonary health and function; low levels of serum 25-hydroxyvitamin $\mathrm{D}_{3}$ are associated with an increased incidence or poor control of asthma, respiratory infection and chronic obstructive pulmonary disease (COPD) ${ }^{1-4}$ Vitamin D is proposed to support respiratory health through promoting antimicrobial functions necessary for efficient clearance of pathogens, and dampening inflammation with the potential to damage lung structure and impair gaseous exchange. ${ }^{4}$ However, the role of its major carrier protein, vitamin D-binding protein (VDBP), is less well understood. A paper by Wood et al in Thorax 5 together with independent studies, highlight the potential of VDBP to influence respiratory function both by determining vitamin $\mathrm{D}$ bioavailability and via direct effects on innate cell function.

$\mathrm{VDBP}$ is a glycosylated $\boldsymbol{\alpha}$-globulin protein synthesised by many tissues including the liver, kidneys, gonads and fat, and also by neutrophils. It binds circulating $25(\mathrm{OH})$-vitamin $\mathrm{D}$ and $1,25(\mathrm{OH})_{2}-$ vitamin $\mathrm{D}$ with high affinity. The levels of this protein far exceed circulating levels of these vitamin $\mathrm{D}$ metabolites, although VDBP has a rapid turnover rate. VDBP has three distinct domains which confer additional functions beyond carriage of vitamin $\mathrm{D}$ (reviewed by Chishimba et $a l^{6}$ ). These functions include augmentation of the monocyte and neutrophil chemotactic response to the complement anaphylotoxin $\mathrm{C} 5 \mathrm{a},{ }^{7} 8$ scavenging of actin molecules released from necrotic cells ${ }^{9}$ and, of interest to the current article, formation of a dimeric molecule with macrophage activating factor (DBP-MAF) which drives macrophages towards a more phagocytic phenotype with increased superoxide

MRC and Asthma UK Centre for Allergic Mechanisms of Asthma, King's College London, London, UK

Correspondence to Catherine Hawrylowicz, MRC and Asthma UK Centre for Allergic Mechanisms of Asthma, King's College London, London SE1 9RT, UK;

catherine.hawrylowicz@kcl.ac.uk generation. $^{10}$ These activities of VDBP may be of particular relevance to the lung environment and, in the case of DBP-MAF formation, especially pertinent in the chronically inflamed lung. The enzymes which promote this dimerisation$\beta$-galactosidase and sialidase-are products of $\mathrm{B}$ and $\mathrm{T}$ lymphocytes, respectively, ${ }^{11}$ cells which occur with increased frequency in airway inflammation and COPD (see, for example, Hogg ${ }^{12}$ ).

The VDBP gene Gc is highly polymorphic. There are three common protein variants-Gc1F, Gc1S and Gc2-and more than 120 rarer variants. ${ }^{13} 14$ These variants have altered affinities for vitamin $\mathrm{D}$ ligands. ${ }^{14} 15$ Gc1F demonstrates greater abundance and affinity for vitamin D metabolites and is better able to maintain serum levels of vitamin $\mathrm{D}$ metabolites. In contrast, Gc2 has lower affinity for vitamin $\mathrm{D}$ metabolites, is less abundant, and also may be less able to convert into DBP-MAF likely to result in reduced macrophage activation. ${ }^{10}$

Findings from genetic association studies of VDBP and respiratory disease are varied and suggest that ethnicity is an important factor. In several small studies in Caucasians the Gc2 protein variant appears protective against $\mathrm{COPD}^{16}{ }^{17}$ while, in some Asian groups, the data suggest that the $\mathrm{Gc1F}$ variant may be a risk factor for the disease. ${ }^{18} 19$ Carriage of the Gc2 variant is also associated with increased susceptibility to active tuberculosis-but this effect was only seen in individuals with severe vitamin $\mathrm{D}$ deficiency, suggesting an interaction between these two factors. ${ }^{20}$ Finally in asthma, while several studies identify a role for vitamin $\mathrm{D}$ status and polymorphisms in the vitamin D receptor, ${ }^{3}$ there are few data to support a role for VDBP, ${ }^{6}$ which may reflect the eosinophilic rather than the neutrophilic nature of this disease in general, although it may be interesting to profile the Gc genotype in severe neutrophilic asthma.

Identification of biological mechanism(s) to explain the various genetic associations of VDBP in different ethnic groups and disease states is challenging. In the present study ${ }^{5}$ the authors try to understand how VDBP may influence the pathology of COPD using a combination of both genetic profiling and in vitro studies. They use clinically well-characterised Caucasian patient populations which include $\alpha_{1}$ antitrypsin-deficient (AATD) individuals with COPD, non-AATD individuals with COPD and healthy non-smoking controls. In these patient populations, and in agreement with some previous studies, ${ }^{6}$ the authors found that the Gc2 variant of VDBP is protective against COPD. However, they also propose that Gc2 is associated with an increased risk of bronchiectasis suggesting-albeit as yet on limited evidence-that this may be due to its reduced capacity to yield DBP-MAF and thereby stimulate macrophages to clear pathogens from the airway.

In addition to profiling the Gc genotype of these individuals, the authors assess the relationship between total serum $25(\mathrm{OH})$ vitamin $\mathrm{D}$, VDBP protein levels and lung function. Their findings confirm some published data of a positive correlation between serum 25(OH)-vitamin $\mathrm{D}$ and forced expiratory volume in $1 \mathrm{~s}\left(\mathrm{FEV}_{1}\right)^{2}$ (reviewed by Chishimba et $a l^{6}$ ) and, in addition, interestingly report an inverse correlation between total serum VDBP and $\mathrm{FEV}_{1}$. However, a lack of association between the Gc genotype and circulating VDBP level makes it difficult to infer from these data how this genotype influences the risk of COPD. Furthermore, it is difficult to dissect the contribution of VDBP from vitamin D itself. Vitamin D metabolites can either enter cells freely by diffusion of unbound vitamin across cell membranes or by endocytosis of that bound to its chaperone VDBP, ${ }^{21}$ although receptor-mediated endocytosis may be less important in macrophages. ${ }^{15}$ It is also possible that high levels of VDBP may be an indirect consequence of low serum 25 $(\mathrm{OH})$-vitamin $\mathrm{D}$ levels which may predispose to respiratory infection; inflammatory cytokines are known to stimulate hepatic transcription of VDBP. ${ }^{22}$

To investigate more directly the immunological activity of VDBP that may be of relevance in COPD, the authors use an innovative in vitro assay to assess alveolar macrophage phagocytic capacity (efferocytosis) by engulfment of fluorescently-labelled apoptotic neutrophils. They show that exogenous VDBP in culture enhanced macrophage activity in a dose-dependent manner, and that sputum from patients with AATD increased macrophage phagocytic activity in a manner that directly correlated with 
the amount of VDBP present. However, anti-DBP only weakly attenuated this observed macrophage activation in vitro. These data suggest that the VDBP component of sputum mediates, at least in part, the increased macrophage activity. Unfortunately, small sample sizes in these in vitro systems meant that it was not possible to discern the influence of different Gc protein variants on macrophage activation and thereby confirm that Gc2 has a reduced capacity to do this, which may have begun to explain its apparent protective role.

Mechanistic studies to underpin epidemiological associations are by their very nature complex and prone to differences in interpretation. Conversely, a second independent study recently suggested that VDBP, in its role as a chaperone for vitamin $\mathrm{D}$, played a pivotal role in determining the bioavailability of vitamin $\mathrm{D}$ to monocytes in vitro. VDBP attenuated vitamin $\mathrm{D}$ metabolite-induced cathelicidin gene expression, demonstrated experimentally by using serum with or without VDBP and, more impressively, by adding back VDBP into monocyte cultures. Importantly, serum from donors with the VDBP genotype Gc2 associated with a lower affinity for vitamin $\mathrm{D}$ metabolites supported greater monocyte responsiveness to vitamin $\mathrm{D}$ than those from Gc1 donors. ${ }^{15}$

The current study by Wood et $a l^{5}$ uses a novel approach to investigate the important question of what role VDBP plays in the pathogenesis of COPD. It provides further support that the Gc2 variant is protective against COPD in Caucasian individuals although, interestingly, it appears to increase the risk of bronchiectasis. The authors also demonstrate directly that serum VDBP levels inversely correlate with lung function in COPD and that sputum VDBP may drive macrophage activation in vitro. It is well known that macrophages accumulate in the lung in COPD, but whether they have a protective role in clearing pathogens and dead cells or cause further damage by the release of inflammatory mediators and neutrophil chemoattractants is not yet well understood. The data in this and additional studies can be used to support both sides of the argument but, on balance, suggest that excessive activation of macrophages by VDBP may do more harm than good. Most importantly, they highlight the need to consider both vitamin $\mathrm{D}$ status and VDBP genotype when investigating the association of vitamin $\mathrm{D}$ with pulmonary function.

\section{Competing interests None.}

Provenance and peer review Commissioned; not externally peer reviewed.

Published Online First 13 January 2011

Thorax 2011:66:189-190

doi:10.1136/thx.2010.154005

\section{REFERENCES}

1. Black PN, Scragg R. Relationship between serum 25-hydroxyvitamin $d$ and pulmonary function in the third national health and nutrition examination survey. Chest 2005;128:3792-8

2. Janssens W, Bouillon R, Claes B, et al. Vitamin D deficiency is highly prevalent in COPD and correlates with variants in the vitamin D-binding gene. Thorax 2010;65:215-20.

3. Litonjua AA, Weiss ST. Is vitamin D deficiency to blame for the asthma epidemic? J Allergy Clin Immunol 2007;120:1031-5

4. Dimeloe S, Nanzer A, Ryanna K, et al. Regulatory T cells, inflammation and the allergic response: the role of glucocorticoids and vitamin D. J Steroid Biochem Mol Biol 2010;120:86-95.

5. Wood AM, Bassford C, Webster D, et al. Vitamin D-binding protein contributed to COPD by activation of alveolar macrophages. Thorax 2011:66:205-10.

6. Chishimba L, Thickett DR, Stockley RA, et al. The vitamin $D$ axis in the lung: a key role for vitamin D-binding protein. Thorax 2010;65:456-62.

7. Piquette CA, Robinson-Hill R, Webster RO. Human monocyte chemotaxis to complement-derived chemotaxins is enhanced by Gc-globulin. J Leukoc Biol 1994;55:349-54.

8. Metcalf JP, Thompson AB, Gossman GL, et al. Gc globulin functions as a cochemotaxin in the lower respiratory tract. A potential mechanism for lung neutrophil recruitment in cigarette smokers. Am Rev Respir Dis 1991;143:844-9.

9. Goldschmidt-Clermont PJ, Allen RC, Nel $A E$, et al. Gc (vitamin D-binding protein) binds the $33.5 \mathrm{~K}$ tryptic fragment of actin. Life Sci 1986;38:735-42.

10. Yamamoto $\mathbf{N}$, Homma S. Vitamin D3 binding protein (group-specific component) is a precursor for the macrophage-activating signal factor from lysophosphatidylcholine-treated lymphocytes. Proc Natl Acad Sci U S A 1991;88:8539-43.

11. Yamamoto $\mathbf{N}$, Kumashiro R. Conversion of vitamin D3 binding protein (group-specific component) to a macrophage activating factor by the stepwise action of beta-galactosidase of $B$ cells and sialidase of T cells. J Immunol 1993;151:2794-802.

12. Hogg JC. Pathophysiology of airflow limitation in chronic obstructive pulmonary disease. Lancet 2004;364:709-21.

13. Cleve H, Constans J. The mutants of the vitamin-Dbinding protein: more than 120 variants of the GC/ DBP system. Vox Sang 1988:54:215-25.

14. Arnaud J, Constans J. Affinity differences for vitamin $D$ metabolites associated with the genetic isoforms of the human serum carrier protein (DBP). Hum Genet 1993:92:183-8.

15. Chun RF, Lauridsen AL, Suon L, et al. Vitamin D-binding protein directs monocyte responses to 25 hydroxy- and 1,25-dihydroxyvitamin D. J Clin Endocrinol Metab 2010;95:3368-76.

16. Horne SL, Cockcroft DW, Dosman JA. Possible protective effect against chronic obstructive airways disease by the GC2 allele. Hum Hered 1990;40:173-6

17. Schellenberg D, Pare PD, Weir TD, et al. Vitamin D binding protein variants and the risk of COPD. Am J Respir Crit Care Med 1998;157:957-61.

18. Ishii T, Keicho N, Teramoto $\mathrm{S}$, et al. Association of Gc-globulin variation with susceptibility to COPD and diffuse panbronchiolitis. Eur Respir $J$ 2001; 18:753-7.

19. Ito I, Nagai S, Hoshino $Y$, et al. Risk and severity of COPD is associated with the group-specific component of serum globulin $1 \mathrm{~F}$ allele. Chest 2004; 125:63-70.

20. Martineau AR, Leandro AC, Anderson ST, et al. Association between Gc genotype and susceptibility to TB is dependent on vitamin D status. Eur Respir $J$ 2010;35:1106-12

21. Nykjaer A, Dragun D, Walther D, et al. An endocytic pathway essential for renal uptake and activation of the steroid $25-(\mathrm{OH})$ vitamin D3. Cell 1999;96:507-15.

22. Guha C, Osawa M, Werner PA, et al. Regulation of human Gc (vitamin D-binding) protein levels: hormonal and cytokine control of gene expression in vitro. Hepatology 1995;21:1675-81. 